\title{
Epicardial adipose tissue: at the heart of the obesity complications
}

\author{
Valeria Guglielmi $^{1,2}$ (D) Paolo Sbraccia ${ }^{1,2}$
}

Received: 29 May 2017/ Accepted: 13 June 2017

(C) Springer-Verlag Italia S.r.l. 2017

\begin{abstract}
In recent years, the anatomic and functional contiguity of epicardial adipose tissue (EAT) to myocardium and coronary arteries has gained increasing interest for its potential pathogenetic role in obesity-related cardiac diseases. Besides its known and attributed biochemical cardioprotective properties, it is becoming evident that, in metabolic disease states, EAT-secreted bioactive molecules may play an important role in the pathogenesis of coronary artery disease and cardiac arrhythmias. EAT-derived inflammatory cytokines and reactive oxidative species may, indeed, play a part in the development of a local proatherogenic milieu by paracrine and vasocrine mechanisms of interaction. In addition, initial clinical and in vitro studies have pointed out that EAT could be a determinant of the substrate of atrial fibrillation by contributing to the structural and electrical remodeling of myocardium. This article reviews the current state of knowledge on the association of EAT with cardiac dysfunction and the potential factors mediating the cross talk between this fat depot and the underlying cardiac structures.
\end{abstract}

Keywords Epicardial adipose tissue - Obesity - Coronary artery disease $\cdot$ Atrial fibrillation $\cdot$ Ectopic fat depots

Managed by Massimo Federici.

Valeria Guglielmi

valeria.guglielmi@uniroma2.it

1 Department of Systems Medicine, University of Rome "Tor Vergata", Via Montpellier 1, 00133 Rome, Italy

2 Internal Medicine Unit and Obesity Center, University Hospital Policlinico Tor Vergata, Rome, Italy

\section{Introduction}

In both developed and developing countries, the prevalence of obesity has reached an alarming level and has become a worldwide epidemic. This has stimulated an enormous research interest in the biology of adipose tissue and in its pathophysiological role in obesity-related complications and therefore gradually transformed adipose tissue from an inert lipid store into a metabolically dynamic endocrine organ capable of synthesizing biologically active compounds that regulate metabolic homeostasis, as well as a variety of biological functions [1-3].

It is well established that abdominal obesity confers increased risk of metabolic complications, whereas preferential fat accumulation in gluteofemoral region is associated with a lower risk [4] and, accordingly to lessons learned from lipodystrophies, may be even protective [5-7]. In addition, there are numerous smaller visceral adipose depots such as epicardial (EAT) and intermuscular adipose tissue [8] that may serve specialized functions related to their neighboring tissues [9]. However, unraveling the mechanisms that differentiate the clinical significance and specific contribution of each fat depot to cardiometabolic complications is challenging since location of different deposits, fundamental properties of adipocytes as well as systemic and local actions of adipokines and FFAs are involved $[10,11]$.

\section{Fat depots around the heart}

The nomenclature used to differentiate fat depots around the heart is often misleading, with several discrepancies and ambiguities among authors [12]. The term of 'paracardial fat' (also known as extrapericardial or intrathoracic) 
has been variably used to identify fat located on the outer surface of the fibrous pericardium [13]. EAT has been instead defined as the intrapericardial fat located between the myocardium and visceral pericardium, while the storage of triglycerides within the cardiomyocytes has been termed myocardial fat [12]. The term 'pericardial fat' is often used interchangeably with EAT, even though some groups used this term as a representative of the total fat around the heart, which is the sum of EAT and paracardial fat [14]. The thickness and volume of EAT and paracardial fat can be quantified by echocardiography and computed tomography or magnetic resonance imaging, respectively $[8,12,15,16]$.

\section{EAT anatomy: what makes plausible the cross talk with myocardium and coronary vessels}

The last decades have witnessed a growing interest in EAT, especially because EAT and myocardium share the same coronary blood supply and are not anatomically separated by any fascial plane, thereby making plausible a 'vasocrine' or 'paracrine' cross talk [9]. In contrast to pericardial fat which derives from the primitive thoracic mesenchyme, EAT originates from splanchnopleuric mesoderm as the omental fat [14].

EAT covers $80 \%$ of the heart's surface, representing $20 \%$ of the organ's total weight under normal conditions, although correlations with advancing age have also been reported $[8,17]$. EAT is mainly found in the atrioventricular and interventricular grooves up to the apex, along the coronary arteries and around the atria.

As compared to other adipose depots, EAT is characterized by smaller adipocytes and greater adipocyte number per gram of tissue, and differences in protein and fatty acid composition have also been reported [18].

\section{EAT physiology: between evidence and speculation}

Unfortunately, since several findings have not been validated in animal models or in human EAT and speciesspecific differences in the physiological relevance of EAT should be expected, the insight into the biology of this unique fat depot remains limited, and further studies aiming at defining the cellular and molecular properties of EAT are still necessary.

However, due to its distinctive anatomic juxtaposition, numerous putative physiological roles have been attributed to EAT. Namely, EAT may attenuate vascular torsion, participate in vessel remodeling and cushion cardiac nervous ganglia; it may also exert metabolic functions representing an immediate energy source in high energy demand conditions and maintaining fatty acid homeostasis in the coronary microcirculation [19]. Indeed, epicardial adipocytes possess special properties to manage fatty acids, including high rates of fatty acid incorporation, synthesis and breakdown as well as insulin-induced lipogenesis [19]. EAT may also act as an immunological tissue protecting cardiac structures against pathogens and inflammatory activators [20] and reduce reactive oxygen species by lowering proton gradients formation [21]. Human EAT has been reported to exhibit high expression of uncoupling protein 1 (UCP1) [22] as well as of genes necessary for inducing browning of white adipose tissue and/or for classical brown adipose tissue development, such as CD137, PR domain containing 16 (PRDM16), peroxisome proliferator-activated receptor gamma coactivator $1 \alpha$ (PGC-1 $\alpha)$, CCAAT/enhancer-binding protein $\alpha(\mathrm{C} / \mathrm{EBP} \alpha)$, and peroxisome proliferator-activated receptor $\gamma(\operatorname{PPAR} \gamma)$ [23]. But whether the presence of UCP1 protein turns into EAT having a higher thermogenic activity remains to be proved. Indeed, in humans undergoing 18fluorodeoxyglucose computed tomography-positron emission tomography fusion scans under experimental cold exposure conditions, the uptake of the tracer (that is the currently accepted criterion of active brown adipose tissue in vivo) is localized in the heart without a clear demarcation between the myocardial and EAT uptake [24]. Although it is becoming apparent that it is a relatively insensitive quantitative technique, the small amount of EAT compared to the much larger, thermogenic and active myocardium makes unlikely that EAT could have a direct thermoregulatory role during chronic cold challenge [23].

EAT may display cardioprotective properties by locally secreting anti-inflammatory and anti-atherogenic adipokines, which are adiponectin and adrenomedullin, whose protein levels correlate with their intracoronary levels $[25,26]$, and omentin that has been shown to ameliorate endothelial function by stimulating endothelial NO synthase and thus the production of nitric oxide [27].

\section{EAT as marker of ectopic fat and metabolic derangements}

EAT mass and thickness are increased in obesity [28], showing a stronger correlation with abdominal fat accumulation [29]. Increased EAT thickness or volume has also been shown to correlate with components of the metabolic syndrome, including fasting plasma glucose and insulin levels, insulin resistance (assessed by euglycemic hyperinsulinemic clamp or surrogate indices like HOMA and QUICKI), atherogenic lipid profile and systolic blood pressure $[30,31]$. On the basis of echocardiographic measurements, definite cutoff values for EAT thickness 
have been even proposed as metabolic syndrome predictors [32]. However, even though a recent meat-analysis supports the relationship between EAT and the metabolic syndrome independent of obesity, the multivariate analysis indicates that the relationship is not robust [33], suggesting that EAT should be mostly regarded as a marker of abdominal adiposity and ectopic fat and lipid storage. Indeed, with regard to the latter, EAT strongly and independently reflects the intramyocardial lipid content, as measured by proton magnetic resonance spectroscopy [34].

However, although the accretion of EAT appears of scarce systemic impact, its local pathophysiological significance has been rapidly growing supported by multiple lines of evidence from basic and translational science as well as from epidemiology.

\section{The clinical relevance of EAT in coronary artery disease}

Despite the above-mentioned associations between EAT amount and abdominal obesity, strong associations between EAT mass and coronary artery disease (CAD) have been found to be independent of measures of body fatness, including intra-abdominal fat [18]. Indeed, EAT volume was independently associated with cardiovascular disease, even after adjusting for body mass index (BMI) and waist circumference [35]. Recently, EAT volume resulted positively and independently associated with coronary artery calcium scoring [36], and able to predict coronary reserve in normal arteries [18]. Besides, nonobese subjects affected by CAD were shown to have higher EAT volume compared to those without CAD [37].

When EAT volume was measured in subjects stratified according to the presence of CAD and obesity, the differences in EAT volume in relation to the presence of CAD were exclusively observed in non-obese patients [37]. In line with other observations $[16,36]$, this suggests that the contribution of EAT to the coronary atherosclerotic burden is more relevant in non-obese than in obese patients and raises the issue of whether obesity may conceal the pathogenetic effects of EAT on CAD progression. Alternatively, it can be theorized that the factors mediating the association between EAT and CAD might change when obesity develops [38].

Instead, whether EAT volume is an indicator for the severity of CAD is still controversial [18].

The direct involvement of EAT in CAD pathogenesis is also sustained by the evidence that intramyocardial segments of coronary arteries, which are, therefore, not in contact with EAT, are not affected by atherosclerosis [39] and that the selective surgical excision of the portion of EAT covering the coronary artery of a pig model of $\mathrm{CAD}$ attenuated the underlying plaque progression in vivo [40].

\section{The association between EAT and CAD from a pathophysiologic perspective}

This epidemiological and experimental evidence together with histopathological data showing the presence of dense inflammatory infiltrates, mainly represented by macrophages, lymphocytes and mast cells, in EAT of CAD patients [41, 42], is the basis for the hypothesis that EAT might locally and detrimentally contribute to coronary atherogenesis.

Innate immunity can be activated within EAT by Tolllike receptors (TLRs) binding, with consequent nuclear factor-kappaB (NFkappaB) translocation into the nucleus inflammatory mediators transcription [42]. As such, EAT sampled from subjects with CAD showed higher NFkappaB, c-Jun N-terminal kinase (JNK) activity, TLR-2 and TLR-4 expression levels, all indicative of macrophage recruitment and activation [42]. Of interest, a recent study using next-generation sequencing technologies demonstrated the presence of bacterial DNA into EAT surrounding diseased coronary arteries, suggesting that EAT is susceptible to microbial colonization that might, thereby, contribute to elicit a proinflammatory response [43].

In CAD patients, EAT expresses and secretes higher levels of proinflammatory markers as compared to subcutaneous fat, such as IL- $1 \beta$, IL- 6 , tumor necrosis factor- $\alpha$ (TNF- $\alpha$ ), monocyte chemoattractant protein-1 (MCP-1), regulated upon activation $\mathrm{T}$ cell and secreted (RANTES) and soluble intercellular adhesion molecules (ICAMs) $[18,41]$, which have been proven to take part in various steps of the atherosclerotic process, from endothelial dysfunction to plaque instability and rupture. In the presence of CAD, elevated expression of IL-6, leptin, resistin, TNF$\alpha$ and visfatin has been also described [18]. In addition, the mRNA levels of secretory type II phospholipase A2 (sPLA2-IIA), an enzyme promoting the generation of more atherogenic low-density lipoproteins and phospholipid products in the artery wall and their retention of in the subendothelial space, have been found increased in EAT from CAD patients [18]. In CAD conditions, as shown by proteomic and secretome analyses, EAT-derived mediators are capable of inducing cell surface expression of adhesion molecules in monocytes and endothelial cells [44], and FFAs released by EAT in the proximity of coronary arteries can modulate vascular responsiveness to vasoactive agents [45]. Contrariwise, EAT anti-inflammatory and anti-atherogenic adipokines (adiponectin and adrenomedullin) are down-regulated in chronic CAD, and increase in intracoronary adrenomedullin levels has been reported only 
when hemodynamic conditions improve as after coronary revascularization $[25,26]$ (Fig. 1).

Furthermore, the expression levels of glucose transporter-4 (GLUT4) are lower, whereas those of renitolbinding protein 4 (RBP4) are higher in EAT from CAD compared to non-CAD subjects [46], suggesting that EAT may partly be responsible for local insulin resistance and altered glucose and lipid profile, which, in turn, can promote atherogenic changes in coronary vessels.

Finally, a higher oxidative stress, which also actively participates in the development and progression of atherosclerotic plaques, has been reported in EAT from CAD patients, as shown by higher levels of reactive oxygen species and lower levels of catalase (an antioxidant enzyme) compared to subcutaneous fat [46]. Recent studies have also reported that the increased oxidative stress characterizing EAT of CAD patients is capable of promoting transdifferentiation of brown to white adipocytes [47] and that an increase in 'brown' features of EAT could predict the stability of coronary atheromas in humans [48]. These data support the hypothesis that the presence of adipocytes with brown features in EAT might also contribute to render the local environment proatherogenic.

The juxtaposition of EAT to myocardium and coronary arteries makes plausible vasocrine (via vasa vasorum) or paracrine (via interstitial fluids) signaling between adipokines and FFAs diffusing from EAT [9, 49]. Besides, cells migrating between these adjacent structures may also contribute to spread inflammation to the surrounding tissues [12].

Although it is so far unclear whether EAT inflammation derives from the underlying plaque inflammation or EAT is intrinsically and primarily inflamed, a bidirectional flow of inflammatory mediators cannot be excluded.

\section{The emerging role of EAT in cardiac arrhythmias}

In obese patients, EAT amount was found to be associated with alterations in cardiac morphology and function such as increased left ventricular mass, right ventricular cavity size, atrial enlargement and diastolic dysfunction [49].

Although these alterations may be due to obesity-induced hemodynamic changes, low-grade inflammation state and oxidative stress $[50,51]$, there is growing evidence of a potential direct role of EAT in their development.

Indeed, several recent studies have established the association of EAT with atrial fibrillation onset, chronicity, recurrence after catheter ablation or electrical cardioversion and symptom burden, as independent of other atrial fibrillation risk factors (i.e., left atrium size) and systemic adiposity [50, 52, 53]. The abundance of EAT independently predicts lone atrial fibrillation, but also of atrial fibrillation associated with hypertrophic cardiomyopathy or CAD and after coronary bypass $[51,54,55]$.

The comparison of the extension and localization of high-dominant frequency and complex fractionated atrial electrograms suggests that EAT might contribute to the formation of the substrate of atrial fibrillation, possibly promoting atrial structural and electrical remodeling [50]. In fact, in line with other fat depots [56, 57], not only extracellular matrix proteins are remarkably increased in EAT in cardiac pathological conditions [58], but EAT may also induce fibrosis in the neighboring myocardium through the secretion of profibrotic mediators including inflammatory cytokines, growth factors and matrix metalloproteinases [59, 60] (Fig. 1). When the secretome of EAT, but not of parasternal subcutaneous fat, obtained from CAD patients was applied on atrial myocardium in an ex vivo model, it induced a transformation of fibroblasts into myofibroblasts and subsequent massive myocardial fibrosis.

Among the EAT-secreted factors, activin A resulted one of the main potential mediators of the profibrotic effect of EAT secretome on atrial myocardium [59]. Of note, activin A concentration in the EAT secretome is highly variable, with higher levels in predisposing conditions for atrial arrhythmias, such as heart failure [59], obesity and type 2 diabetes [60]. Of note, type 2 diabetes-induced alterations in the secretory profile of EAT have been even overall associated with the development of insulin resistance and negative inotropic effects in rat cardiomyocytes, suggesting that in type 2 diabetes EAT could also locally contribute to the pathogenesis of cardiomyopathy [60].

However, both the abundance of EAT at sites which are common targets for atrial fibrillation catheter ablation (i.e., at the antra of pulmonary veins) and the dense autonomic innervation of EAT may suggest that EAT could act as a trigger and a modulator of the sympathetic and parasympathetic tone in arrhythmias [50]. After all, EAT is a source of inflammatory cytokines and reactive oxygen species, known to be associated with a high incidence of atrial fibrillation, as well as of adipokines involved in calcium homeostasis of cardiomyocytes and, therefore, in the electrical properties of the atria [60].

Finally, the presence of marked myocardial fibro-fatty infiltration in cardiac disorders strictly associated with the development of ventricular arrhythmias, such as the arrhythmogenic right ventricular cardiomyopathy and the myotonic dystrophy, as well as in an ovine model of ischemic cardiomyopathy [61], suggests that EAT may also play a role in the pathogenesis of ventricular arrhythmias. 


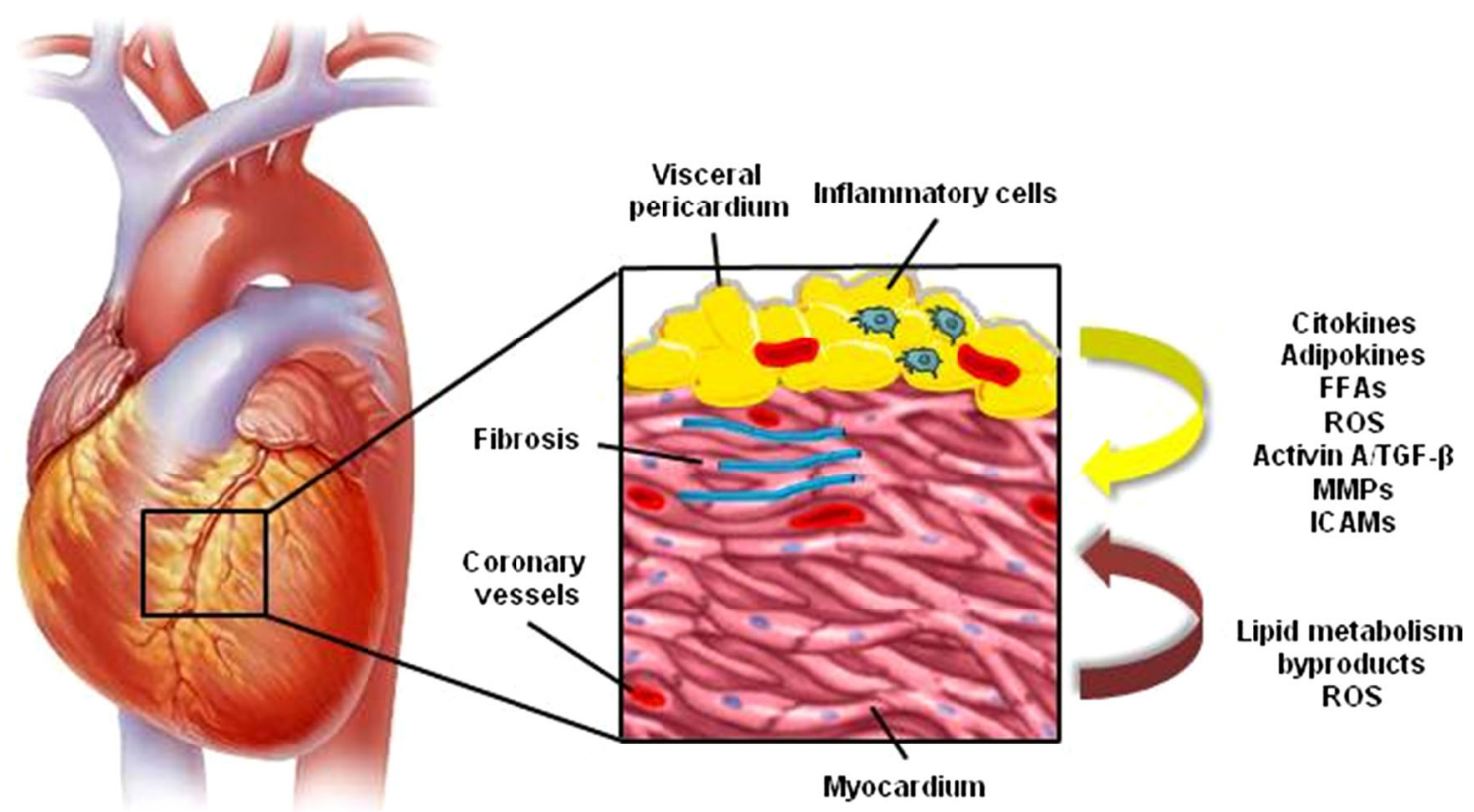

Fig. 1 Potential signaling pathways mediating the cross talk between EAT, myocardium and coronary vessels

\section{Does EAT location matter?}

Necropsic and multidetector computed tomography studies have shown that EAT is not homogeneously distributed and reported regional variation in the strengths of associations between different measurements of EAT and anthropometric indexes [16]. Similarly, EAT thickness measured at the level of the left atrioventricular groove, but not over the right ventricular free wall, was related to clustering of metabolic syndrome components and inflammatory markers [16], whereas the peri-atrial EAT volume resulted the most strictly associated with the incidence atrial fibrillation [62].

At molecular level, Spiroglou et al. [63] reported different patterns of adipokine expression (adiponectin, visfatin, leptin, chemerin, and vaspin) between peri-coronary and apical EAT, failing to find any correlation between coronary atherosclerosis and adipokine expression in EAT of the apical region.

A recent study, by using a pangenomic approach, even defined specific transcriptomic signatures of EAT at different anatomical locations as suggestive of different EAT properties with respect to the adjacent structures. In particular, the peri-coronary EAT resulted more closely involved in atherotic plaques formation, expressing genes involved in cell proliferation and in metabolism of sphingolipids which are lipid components of atheromas capable of promoting oxidized LDL and VLDL retention in the sub-endothelial space. In contrast, peri-ventricular EAT was more strictly linked to genes regulating inflammatory pathways, whereas the genes most up-regulated by periatrial EAT were implicated in energy metabolism, cardiomyocyte contractility and intracellular calcium signaling [64].

\section{Therapeutic interventions}

Changes in nutritional $[65,66]$ or physical activity $[67,68]$, and more recently bariatric surgery [69-71], are the mainstay interventions for obese and type 2 diabetic patients. In this context, EAT has been discussed as a target for the treatment and prevention of obesity cardiovascular complications.

Weight loss following dietary as well as bariatric surgery interventions was significantly associated with reductions in EAT, whereas the impact of physical exercise on EAT amount is less established [72]. The effect on EAT by medications has been also evaluated. Park et al. [73] reported a significant decrease in EAT thickness in patients treated with atorvastatin, whereas it increased during pioglitazone treatment [74].

However, the amount of EAT reduction cannot be predicted by the overall amount of weight loss, as most impressively demonstrated by the finding that, in spite of the much greater reduction in body weight occurred by means of bariatric surgery, EAT decrease was similar to that observed in the group of patients undergone dietary intervention [72]. This could suggest that there is a limit on the possible EAT reduction in humans consistently with the 
need for EAT to retain its physiologic role, but also that EAT responses to weight loss strategies may vary from those of larger visceral fat depots.

All together, these observations highlight that additional epidemiological and experimental research is needed not only to provide evidence of a definite causal relationship between EAT and obesity-related cardiac dysfunctions and to unravel mechanistically their link, but also to investigate the beneficial effect of therapeutic interventions aiming at EAT reduction.

\section{Compliance with ethical standards}

Conflict of interest The authors declare that they have no conflict of interest.

Human and animal rights This article does not contain any studies with human or animal subjects performed by the any of the authors.

Informed consent For this type of study formal consent is not required.

\section{References}

1. Tilg H, Moschen AR (2006) Adipocytokines: mediators linking adipose tissue, inflammation and immunity. Nat Rev Immunol 6(10):772-783. doi:10.1038/nri1937

2. Coelho M, Oliveira T, Fernandes R (2013) Biochemistry of adipose tissue: an endocrine organ. Arch Med Sci 9(2):191-200. doi:10.5114/aoms.2013.3318120246

3. Guglielmi V, D'Adamo M, Bellia A et al (2015) Iron status in obesity: an independent association with metabolic parameters and effect of weight loss. Nutr Metab Cardiovasc Dis 25(6):541-547. doi:10.1016/j.numecd.2015.02.012S0939-4753(15)00055-1

4. Marucci A, Menzaghi C, Copetti M et al (2015) Strong evidence of sexual dimorphic effect of adiposity excess on insulin sensitivity. Acta Diabetol 52(5):991-998. doi:10.1007/s00592-0150804-2

5. Coutinho T, Goel K, Correa de Sa D et al (2011) Central obesity and survival in subjects with coronary artery disease: a systematic review of the literature and collaborative analysis with individual subject data. J Am Coll Cardiol 57(19):1877-1886. doi:10.1016/j. jacc.2010.11.058S0735-1097(11)00732-7

6. Lombardi F, Gullotta F, Columbaro M et al (2007) Compound heterozygosity for mutations in LMNA in a patient with a myopathic and lipodystrophic mandibuloacral dysplasia type A phenotype. J Clin Endocrinol Metab 92(11):4467-4471. doi:10. 1210/jc.2007-0116

7. Guglielmi V, D'Adamo M, D'Apice MR et al (2010) Elbow deformities in a patient with mandibuloacral dysplasia type A. Am J Med Genet A 152A(11):2711-2713. doi:10.1002/ajmg.a.33700

8. Guglielmi V, Maresca L, D'Adamo M et al (2014) Age-related different relationships between ectopic adipose tissues and measures of central obesity in sedentary subjects. PLoS ONE 9(7):e103381. doi:10.1371/journal.pone.0103381PONE-D-14-22290

9. Sacks HS, Fain JN (2007) Human epicardial adipose tissue: a review. Am Heart J 153(6):907-917. doi:10.1016/j.ahj.2007.03. 019

10. Kwok KH, Lam KS, Xu A (2016) Heterogeneity of white adipose tissue: molecular basis and clinical implications. Exp Mol Med 48:e215. doi:10.1038/emm.2016.5emm20165
11. Morano S, Romagnoli E, Filardi T et al (2015) Short-term effects of glucagon-like peptide 1 (GLP-1) receptor agonists on fat distribution in patients with type 2 diabetes mellitus: an ultrasonography study. Acta Diabetol 52(4):727-732. doi:10.1007/ s00592-014-0710-z

12. Iozzo P (2011) Myocardial, perivascular, and epicardial fat. Diabetes Care 34(Suppl 2):S371-S379. doi:10.2337/dc11s25034/Supplement_2/S371

13. Bertaso AG, Bertol D, Duncan BB, Foppa M (2013) Epicardial fat: definition, measurements and systematic review of main outcomes. Arq Bras Cardiol 101(1):e18-e28. doi:10.5935/abc. 20130138S0066-782X2013002700020

14. Iacobellis G (2009) Epicardial and pericardial fat: close, but very different. Obesity (Silver Spring) 17(4):625. doi:10.1038/oby. 2008.575oby2008575 (author reply 626-627)

15. Iacobellis G, Willens HJ (2009) Echocardiographic epicardial fat: a review of research and clinical applications. J Am Soc Echocardiogr 22(12):1311-1319. doi:10.1016/j.echo.2009.10. 013S0894-7317(09)00996-1 (quiz 1417-1318)

16. Gorter PM, van Lindert AS, de Vos AM et al (2008) Quantification of epicardial and peri-coronary fat using cardiac computed tomography; reproducibility and relation with obesity and metabolic syndrome in patients suspected of coronary artery disease. Atherosclerosis 197(2):896-903. doi:10.1016/j.atherosclerosis. 2007.08.016

17. Schejbal V (1989) Epicardial fatty tissue of the right ventriclemorphology, morphometry and functional significance. Pneumologie 43(9):490-499

18. Ouwens DM, Sell H, Greulich S, Eckel J (2010) The role of epicardial and perivascular adipose tissue in the pathophysiology of cardiovascular disease. J Cell Mol Med 14(9):2223-2234. doi:10.1111/j.1582-4934.2010.01141.xJCMM1141

19. Marchington JM, Pond CM (1990) Site-specific properties of pericardial and epicardial adipose tissue: the effects of insulin and high-fat feeding on lipogenesis and the incorporation of fatty acids in vitro. Int J Obes 14(12):1013-1022

20. Fain JN, Sacks HS, Bahouth SW, Tichansky DS, Madan AK, Cheema PS (2010) Human epicardial adipokine messenger RNAs: comparisons of their expression in substernal, subcutaneous, and omental fat. Metabolism 59(9):1379-1386. doi:10. 1016/j.metabol.2009.12.027S0026-0495(09)00546-0

21. Sacks HS, Fain JN (2011) Human epicardial fat: what is new and what is missing? Clin Exp Pharmacol Physiol 38(12):879-887. doi:10.1111/j.1440-1681.2011.05601.x

22. Chechi K, Blanchard PG, Mathieu P, Deshaies Y, Richard D (2013) Brown fat like gene expression in the epicardial fat depot correlates with circulating HDL-cholesterol and triglycerides in patients with coronary artery disease. Int $\mathrm{J}$ Cardiol 167(5):2264-2270. doi:10.1016/j.ijcard.2012.06.008S01675273(12)00783-8

23. Sacks HS, Fain JN, Bahouth SW et al (2013) Adult epicardial fat exhibits beige features. J Clin Endocrinol Metab 98(9):E1448E1455. doi:10.1210/jc.2013-1265jc.2013-1265

24. Vijgen GH, Bouvy ND, Teule GJ et al (2012) Increase in brown adipose tissue activity after weight loss in morbidly obese subjects. J Clin Endocrinol Metab 97(7):E1229-E1233. doi:10.1210/ jc.2012-1289jc.2012-1289

25. Iacobellis G, di Gioia CR, Di Vito M et al (2009) Epicardial adipose tissue and intracoronary adrenomedullin levels in coronary artery disease. Horm Metab Res 41(12):855-860. doi:10. 1055/s-0029-1231081

26. Iacobellis G, di Gioia CR, Cotesta D et al (2009) Epicardial adipose tissue adiponectin expression is related to intracoronary adiponectin levels. Horm Metab Res 41(3):227-231. doi:10.1055/ s-0028-1100412 
27. Fain JN, Sacks HS, Buehrer B et al (2008) Identification of omentin mRNA in human epicardial adipose tissue: comparison to omentin in subcutaneous, internal mammary artery periadventitial and visceral abdominal depots. Int $\mathrm{J}$ Obes (Lond) 32(5):810-815. doi:10.1038/sj.ijo.08037900803790

28. Cikim AS, Topal E, Harputluoglu M et al (2007) Epicardial adipose tissue, hepatic steatosis and obesity. $\mathrm{J}$ Endocrinol Invest 30(6):459-464. doi:10.1007/BF03346328

29. Iacobellis G, Ribaudo MC, Assael F et al (2003) Echocardiographic epicardial adipose tissue is related to anthropometric and clinical parameters of metabolic syndrome: a new indicator of cardiovascular risk. J Clin Endocrinol Metab 88(11):5163-5168. doi:10.1210/jc.2003-030698

30. Iacobellis G, Barbaro G, Gerstein HC (2008) Relationship of epicardial fat thickness and fasting glucose. Int J Cardiol 128(3):424-426. doi:10.1016/j.ijcard.2007.12.072S0167-5273(08)00127-7

31. Wang CP, Hsu HL, Hung WC et al (2009) Increased epicardial adipose tissue (EAT) volume in type 2 diabetes mellitus and association with metabolic syndrome and severity of coronary atherosclerosis. Clin Endocrinol (Oxf) 70(6):876-882. doi:10. 1111/j.1365-2265.2008.03411.xCEN3411

32. Iacobellis G, Willens HJ, Barbaro G, Sharma AM (2008) Threshold values of high-risk echocardiographic epicardial fat thickness. Obesity (Silver Spring) 16(4):887-892. doi:10.1038/ oby.2008.6oby 20086

33. Rabkin SW (2014) The relationship between epicardial fat and indices of obesity and the metabolic syndrome: a systematic review and meta-analysis. Metab Syndr Relat Disord 12(1):31-42. doi:10.1089/met.2013.0107

34. Malavazos AE, Di Leo G, Secchi F et al (2010) Relation of echocardiographic epicardial fat thickness and myocardial fat. Am J Cardiol 105(12):1831-1835. doi:10.1016/j.amjcard.2010. 01.368S0002-9149(10)00527-8

35. Mahabadi AA, Massaro JM, Rosito GA et al (2009) Association of pericardial fat, intrathoracic fat, and visceral abdominal fat with cardiovascular disease burden: the Framingham Heart Study. Eur Heart J 30(7):850-856. doi:10.1093/eurheartj/ ehn573ehn573

36. Bettencourt N, Toschke AM, Leite D et al (2012) Epicardial adipose tissue is an independent predictor of coronary atherosclerotic burden. Int J Cardiol 158(1):26-32. doi:10.1016/j. ijcard.2010.12.085S0167-5273(10)01145-9

37. Iwayama T, Nitobe J, Watanabe T et al (2014) Role of epicardial adipose tissue in coronary artery disease in non-obese patients. J Cardiol 63(5):344-349. doi:10.1016/j.jjcc.2013.10.002S09145087(13)00301-8

38. Strissel KJ, Denis GV, Nikolajczyk BS (2014) Immune regulators of inflammation in obesity-associated type 2 diabetes and coronary artery disease. Curr Opin Endocrinol Diabetes Obes 21(5):330-338. doi:10.1097/MED.0000000000000085

39. Greif M, Becker A, von Ziegler F et al (2009) Pericardial adipose tissue determined by dual source $\mathrm{CT}$ is a risk factor for coronary atherosclerosis. Arterioscler Thromb Vasc Biol 29(5):781-786. doi:10.1161/ATVBAHA.108.180653ATVBAHA.108.180653

40. McKenney ML, Schultz KA, Boyd JH et al (2014) Epicardial adipose excision slows the progression of porcine coronary atherosclerosis. J Cardiothorac Surg 9:2. doi:10.1186/1749-80909-21749-8090-9-2

41. Mazurek T, Zhang L, Zalewski A et al (2003) Human epicardial adipose tissue is a source of inflammatory mediators. Circulation 108(20):2460-2466. doi:10.1161/01.CIR.0000099542.57313. C501.CIR.0000099542.57313.C5

42. Baker AR, Harte AL, Howell N et al (2009) Epicardial adipose tissue as a source of nuclear factor-kappaB and c-Jun N-terminal kinase mediated inflammation in patients with coronary artery disease. J Clin Endocrinol Metab 94(1):261-267. doi:10.1210/jc. 2007-2579jc.2007-2579

43. Pedicino D, Severino A, Ucci S et al (2017) Epicardial adipose tissue microbial colonization and inflammasome activation in acute coronary syndrome. Int J Cardiol 236:95-99. doi:10.1016/j. ijcard.2017.02.040

44. Karastergiou K, Fried SK (2013) Multiple adipose depots increase cardiovascular risk via local and systemic effects. Curr Atheroscler Rep 15(10):361. doi:10.1007/s11883-013-0361-5

45. Henrichot E, Juge-Aubry CE, Pernin A et al (2005) Production of chemokines by perivascular adipose tissue: a role in the pathogenesis of atherosclerosis? Arterioscler Thromb Vasc Biol 25(12):2594-2599. doi:10.1161/01.ATV.0000188508.40052.35

46. Salgado-Somoza A, Teijeira-Fernandez E, Rubio J, Couso E, Gonzalez-Juanatey JR, Eiras S (2012) Coronary artery disease is associated with higher epicardial retinol-binding protein 4 (RBP4) and lower glucose transporter (GLUT) 4 levels in epicardial and subcutaneous adipose tissue. Clin Endocrinol (Oxf) 76(1):51-58. doi:10.1111/j.1365-2265.2011.04140.x

47. Dozio E, Vianello E, Briganti S et al (2014) Increased reactive oxygen species production in epicardial adipose tissues from coronary artery disease patients is associated with brown-to-white adipocyte trans-differentiation. Int J Cardiol 174(2):413-414. doi:10.1016/j.ijcard.2014.04.045S0167-5273(14)00704-9

48. Ahmadi N, Nabavi V, Hajsadeghi F et al (2013) Aged garlic extract with supplement is associated with increase in brown adipose, decrease in white adipose tissue and predict lack of progression in coronary atherosclerosis. Int J Cardiol 168(3):2310-2314. doi:10. 1016/j.ijcard.2013.01.182S0167-5273(13)00242-8

49. Iacobellis G, Bianco AC (2011) Epicardial adipose tissue: emerging physiological, pathophysiological and clinical features. Trends Endocrinol Metab 22(11):450-457. doi:10.1016/j.tem. 2011.07.003S1043-2760(11)00112-3

50. Hatem SN, Redheuil A, Gandjbakhch E (2016) Cardiac adipose tissue and atrial fibrillation: the perils of adiposity. Cardiovasc Res 109(4):502-509. doi:10.1093/cvr/cvw001cvw001

51. Guglielmi V, Maresca L, Lanzillo C et al (2016) Relationship between regional fat distribution and hypertrophic cardiomyopathy phenotype. PLoS ONE 11(7):e0158892. doi:10.1371/ journal.pone.0158892PONE-D-16-00255

52. Tsao HM, Hu WC, Wu MH et al (2011) Quantitative analysis of quantity and distribution of epicardial adipose tissue surrounding the left atrium in patients with atrial fibrillation and effect of recurrence after ablation. Am J Cardiol 107(10):1498-1503. doi:10.1016/j.amjcard.2011.01.027S0002-9149(11)00339-0

53. Nagashima K, Okumura Y, Watanabe I et al (2011) Association between epicardial adipose tissue volumes on 3-dimensional reconstructed CT images and recurrence of atrial fibrillation after catheter ablation. Circ J 75(11):2559-2565. doi:10.1253/circj.CJ11-0554

54. Muhib S, Fujino T, Sato N, Hasebe N (2013) Epicardial adipose tissue is associated with prevalent atrial fibrillation in patients with hypertrophic cardiomyopathy. Int Heart J 54(5):297-303. doi:10.1536/ihj.54.297

55. Opolski MP, Staruch AD, Kusmierczyk M et al (2015) Computed tomography angiography for prediction of atrial fibrillation after coronary artery bypass grafting: proof of concept. J Cardiol 65(4): 285-292. doi:10.1016/j.jjcc.2014.12.006S0914-5087(14)00359-1

56. Guglielmi V, Cardellini M, Cinti F et al (2015) Omental adipose tissue fibrosis and insulin resistance in severe obesity. Nutr Diabetes 5:e175. doi:10.1038/nutd.2015.22nutd201522

57. Cancello R, Tordjman J, Poitou C et al (2006) Increased infiltration of macrophages in omental adipose tissue is associated with marked hepatic lesions in morbid human obesity. Diabetes 55(6):1554-1561. doi:10.2337/db06-0133 
58. Jiang DS, Zeng HL, Li R et al (2017) Aberrant epicardial adipose tissue extracellular matrix remodeling in patients with severe ischemic cardiomyopathy: insight from comparative quantitative proteomics. Sci Rep 7:43787. doi:10.1038/srep43787srep43787

59. Venteclef N, Guglielmi V, Balse E et al (2015) Human epicardial adipose tissue induces fibrosis of the atrial myocardium through the secretion of adipo-fibrokines. Eur Heart J 36(13):795-805a. doi:10.1093/eurheartj/eht099eht099

60. Greulich S, Maxhera B, Vandenplas G et al (2012) Secretory products from epicardial adipose tissue of patients with type 2 diabetes mellitus induce cardiomyocyte dysfunction. Circulation 126(19):2324-2334. doi:10.1161/CIRCULATIONAHA.111. 039586CIRCULATIONAHA. 111.039586

61. Samanta R, Pouliopoulos J, Thiagalingam A, Kovoor P (2016) Role of adipose tissue in the pathogenesis of cardiac arrhythmias. Heart Rhythm 13(1):311-320. doi:10.1016/j.hrthm.2015.08. 016S1547-5271(15)01030-9

62. Nakanishi K, Fukuda S, Tanaka A et al (2012) Peri-atrial epicardial adipose tissue is associated with new-onset nonvalvular atrial fibrillation. Circ J 76(12):2748-2754. doi:10.1253/circj.CJ-12-0637

63. Spiroglou SG, Kostopoulos CG, Varakis JN, Papadaki HH (2010) Adipokines in periaortic and epicardial adipose tissue: differential expression and relation to atherosclerosis. J Atheroscler Thromb 17(2):115-130. doi:10.5551/jat.1735

64. Gaborit B, Venteclef N, Ancel P et al (2015) Human epicardial adipose tissue has a specific transcriptomic signature depending on its anatomical peri-atrial, peri-ventricular, or peri-coronary location. Cardiovasc Res 108(1):62-73. doi:10.1093/cvr/cvv208cvv208

65. Sacks FM, Bray GA, Carey VJ et al (2009) Comparison of weight-loss diets with different compositions of fat, protein, and carbohydrates. N Engl J Med 360(9):859-873. doi:10.1056/NEJ Moa0804748360/9/859

66. Lombardo M, Bellia A, Padua E et al (2014) Morning meal more efficient for fat loss in a 3-month lifestyle intervention. J Am Coll Nutr 33(3):198-205. doi:10.1080/07315724.2013.863169
67. Bellia A, Salli M, Lombardo M et al (2014) Effects of whole body vibration plus diet on insulin-resistance in middle-aged obese subjects. Int J Sports Med 35(6):511-516. doi:10.1055/s0033-1354358

68. Jakicic JM, Otto AD (2005) Physical activity considerations for the treatment and prevention of obesity. Am J Clin Nutr 82(1 Suppl):226S-229S

69. Lombardo M, Bellia A, Mattiuzzo F et al (2015) Frequent followup visits reduce weight regain in long-term management after bariatric surgery. Bariatr Surg Pract Patient Care 10(3):119-125. doi:10.1089/bari.2015.0021

70. Frikke-Schmidt H, O'Rourke RW, Lumeng CN, Sandoval DA, Seeley RJ (2016) Does bariatric surgery improve adipose tissue function? Obes Rev 17(9):795-809. doi:10.1111/obr.12429

71. Hansen M, Lund MT, Jorgensen AL et al (2016) The effects of diet- and RYGB-induced weight loss on insulin sensitivity in obese patients with and without type 2 diabetes. Acta Diabetol 53(3):423-432. doi:10.1007/s00592-015-0812-210.1007/s00592015-0812-2

72. Rabkin SW, Campbell H (2015) Comparison of reducing epicardial fat by exercise, diet or bariatric surgery weight loss strategies: a systematic review and meta-analysis. Obes Rev 16(5):406-415. doi:10.1111/obr.12270

73. Park JH, Park YS, Kim YJ et al (2010) Effects of statins on the epicardial fat thickness in patients with coronary artery stenosis underwent percutaneous coronary intervention: comparison of atorvastatin with simvastatin/ezetimibe. J Cardiovasc Ultrasound 18(4):121-126. doi:10.4250/jcu.2010.18.4.121

74. Jonker JT, Lamb HJ, van der Meer RW et al (2010) Pioglitazone compared with metformin increases pericardial fat volume in patients with type 2 diabetes mellitus. J Clin Endocrinol Metab 95(1):456-460. doi:10.1210/jc.2009-1441jc.2009-1441 